\title{
Comparative Analysis of Group Effect and Individual Effect Under Negative Emotion
}

\author{
Zhaoyu Wang* \\ Zhengzhou foreign language school, Zhengzhou, 450001, China, \\ ${ }^{*}$ Corresponding author. Email: Wzy3956@outlook.com
}

\begin{abstract}
Compared with individuals experiencing emotional events alone when individuals share the same emotional event with others and share the same emotion. When one points to the emotional state of the target, the individual's emotional experience will be enhanced. We define this phenomenon as group. Amplification effect of body emotion. This study investigated the amplification effect of group emotion through two experiments. The "evaluation confirmation amplification" model explains this phenomenon theoretically.

The results show that there are significant differences in the emotional scores of "anger and hatred" in the three situations, and the "anger and hatred" in the anger confirmation group is significantly higher than that in the single group. The occurrence of emotional "confirmation" process depends on whether individuals can realize and recognize the emotional state of others. Only when individual emotions are confirmed by others, their emotional experience will be enhanced, that is, the amplification effect of group emotions will be produced. The results of this study verify the amplification effect of group emotion to a certain extent, and preliminarily explore its generation mechanism.
\end{abstract}

Keywords: Group size, Group emotion, confirm

\section{INTRODUCTION}

"Emotion is a general term for a series of subjective cognitive experiences, an organized, profound and constantly changing psychological state." It is an individual's view and evaluation of an event, and a purposeful, pluralistic, and complex social expression. Arnold Lazarus's cognitive evaluation theory regards emotion as the product of the interaction between man and the environment. In emotional activities, individuals should not only accept the impact of stimulus events in the environment on themselves, evaluate the relationship between stimulus events and themselves, but also adjust their response to stimuli. This theory emphasizes that cognitive evaluation plays a very important role in the process of stress response. Cognitive activities play a guiding role. Only by forming correct cognition can we accurately grasp the significance of stimulating events, and then emotional activities can choose appropriate and effective action combinations, namely action response.

\section{AMPLIFICATION EFFECT OF GROUP EMOTION}

\subsection{Amplification effect of group emotion}

Some previous studies have shown that in some specific social situations, individual emotional experience will be amplified. For example, Jackbs, man stead and Fisher [1] created four different story situations (pleasure, sadness, anxiety and anger) in text form. Taking the pleasant situation as an example, one experimental condition is that the subjects imagine that they have won the prize unexpectedly; Under the other four conditions, subjects imagined experiencing emotional events with a friend and manipulated the role of friends: whether they expressed corresponding emotions as a co experiencer or observer. In the story situation of "common experience", the subjects imagined that "they bought a lottery ticket with their friends and won the prize".

In the story situation of "Observer", the subjects imagined that "they won the prize and happened to be with their friends"; In the story situation of "expressing emotion", describe his friend's "very enthusiastic 
response", and in the story situation of "not expressing emotion", describe his friend's "very calm and commented that he could have won a bigger prize". The results showed that when experiencing pleasure events with others (winning the prize with friends) and others express pleasure emotions, individuals experience stronger pleasure emotions; When friends express sadness (common friend dies), individuals experience stronger sadness.

In another study, Fischer, man stead, Evers and over [2] asked subjects to imagine that they and their classmates worked hard to write a paper, but they were rated low by the teacher - sharing emotional events, and then manipulating the emotional responses of other students in the class in the form of text presentation. The anger situation is described as "the classroom atmosphere is terrible.

All the students looked angry "; The sad situation is described as "the classroom atmosphere is very depressed, the students are obviously disappointed, and all the students look very sad". Then ask the subjects to answer questions to show how they will react emotionally in this situation. The results showed that when others expressed anger, subjects reported experiencing stronger anger; When others expressed sadness, subjects experienced stronger sadness. Although these studies do not involve the concept of "sharing", they imply that sharing emotional events will amplify individual emotional experience.

\subsection{Mechanism of amplification effect of group emotion}

According to the theory of emotional cognitive evaluation, the generation of individual emotion is the result of individual evaluation of emotional stimulation and stimulation situation. When individuals share emotional events with others, individuals will evaluate the emotional information of others.

The evaluation process is more likely to occur in fuzzy and uncertain social situations. Individuals pay more attention to the emotional information of others, to reduce the uncertainty of their emotional state. Due to the complex attributes of emotion itself and the subjectivity of individual emotional experience itself, what is induced by emotional events in real life is often not a single and definite emotion, but more fuzzy composite emotions (such as shame, guilt) or mixed emotions such as anger and sadness. In short, the emotional experience induced by emotional situations (including stress and threat situations) in real life is basically vague and uncertain. When individuals share emotional events with others, others' emotional response provides important social information for individual social assessment, so individuals will pay more attention to others' emotional information when conducting social assessment.
The "evaluation confirmation amplification" model is summarized as follows: when individuals share emotional events with other members of the group

Emotional events will first induce the individual's emotional preparation state. Because the individual's subjective emotional experience itself is fuzzy, the individual will evaluate the emotional response of others and integrate the emotional information of others to clarify his own emotional state and reduce cognitive uncertainty. Because of sharing emotional events, the emotional reactions of individuals and other members of the group are often consistent. Therefore, the individual's emotions can also be confirmed by other members of the group. If the individual's emotion is confirmed by others, especially in the group, the individual's emotion is repeatedly recognized and confirmed by other members, then the subjective and uncertain individual emotion will become an objective and stable "shared emotion". Individuals will be surer of their emotions, so individual emotional experience can be strengthened, resulting in the amplification effect of group emotionsv[3]. On the contrary, if the individual's emotion is not confirmed by other members of the group, the individual's emotion is still subjective and unstable, and the individual will question the rationality of his emotion and even inhibit his emotion. Therefore, the individual's emotion will not be amplified in the group situation.

\section{TEST THE "EVALUATION CONFIRMATION AMPLIFICATION" MODEL}

\subsection{Purpose and hypothesis of the experiment}

Experiment 2 intends to test the "evaluation confirmation amplification" model by setting false subjects to control other people's emotional reactions and manipulating the "confirmation" conditions. According to our model theory, individuals share emotional events with others in the group, and then evaluate the emotional response of others - infer the inner emotional state of others through the external behavior of others [4]. If individuals find that the emotions of others are consistent with their own, their emotions will be confirmed and individuals will obtain stable and reliable psychological support, Will more determine their own emotional experience, and the individual emotions in the group situation will be amplified. In addition, someone believe that individuals experiencing emotional stimuli with others will allocate more cognitive resources to emotional stimuli, resulting in the enhancement of individual emotional experience. If joint attention makes subjects pay more attention to emotional video, resulting in group sharing emotional amplification effect, then subjects in group will pay more attention to video details than those in single 
group. We believe that the evaluation confirmed that it led to the amplification of group emotions rather than common concern.

The hypotheses of this study are: (1) when others express their emotions clearly, individual emotions are confirmed by others, resulting in the amplification effect of group emotions;(2) There was no significant difference in subjects' attention to video under the three situations.

\subsection{Experimental method}

Subjects90 students from Ningbo University were randomly selected, and the subjects were required to have not done the experiment of video evaluation before.

There are 18 boys and 72 girls, with an average age of 18.60. After the experiment, each subject will receive a small gift.

When the degree is 10, mark "' " at the corresponding position in the transverse direction. The more left, the weaker the emotional state, the more right

Indicates that the stronger the emotional state.

The subjects were randomly assigned to three situations: single situation group and anger confirmation situation

There were 30 subjects in each group. Each situation condition and the gender ratio in the group are basically the same. Since it is necessary to control the emotional response of others and ensure that individuals can recognize the emotional response of others - emotional confirmation when sharing emotional events with others, a false subject of both sexes is set to express their emotional response. According to the evaluation of preexperimental emotional video materials, the two highest emotions induced by video are anger and emotion

Sadness, so set up anger confirmation situation and sadness confirmation situation. Anger confirmation situation refers to that subjects watch videos with two false subjects, and the false subjects express their anger; Sadness confirmation situation refers to that the subjects watch the video together with two false subjects, and the false subjects express their sadness. The subjects in the single situation group watched the video themselves. In addition, we also carried out expression training for false subjects. Angry facial expressions require frowning and pupil dilation; When you speak your lines, you speak faster, with tension and ups and downs in tone. Sad facial expressions require eyebrows to rise, eyebrows to remain depressed as a whole, lips to be closed, eyes to be dim, etc. speak slowly. After confirming the details of all aspects with the false subjects, play the video to let the false subjects train by themselves. After being proficient, let the false subjects practice, record the exercise process with a camera, and
Practice for many times until both the main test and the false subjects think that they can naturally and truly express their anger or sadness.

\subsection{Result analysis}

In the anger confirmation situation group and sadness confirmation situation group, we set the questionnaire title "do you think you are watching

What emotions did the other two subjects experience most during the video? The option is "subjects 2 and 3 mainly experience: pleasure, anger, sadness and disappointment", which is used to test whether the emotional expression of our two false subjects is effective, that is, whether the subjects are aware of and accurately identify the emotional response of the false subjects (false subjects' express anger in the anger confirmation situation group and sadness in the sadness confirmation situation group). After data statistics (see attached Fig 1), the results showed that in the anger confirmation situation group, the accurate judgment rate of anger of false subject No. 3 was 0.82 , and that of false subject No. 2 was 0.69 . In addition, 16 of the 28 subjects in the anger confirmation situation group felt that two false subjects experienced anger at the same time, and the accurate judgment rate was 0.57 . In the sadness confirmation situation group, the correct judgment rate was 0.57 The accurate judgment rate of No. 3 sadness was 0.67 , and the accurate judgment rate of No. 2 false subject was 0.33 . In addition, 8 of the 30 subjects felt that two false subjects experienced sadness at the same time, and the accurate judgment rate was 0.27 . The results showed that our operation of response to others' anger was effective $(0.82,0.69$ and 0.57 were greater than the theoretical median value of 0.5 ), two false subjects naturally and truly expressed their anger, and the subjects also perceived the anger response of others. However, in the sadness confirmation situation, only most subjects felt that false subject 3 experienced sadness $(0.82$ was greater than the theoretical median of 0.5 , but 0.33 and 0.27 were less than the theoretical median of 0.5 ), the sad emotion response of false subject 2 was not accurately recognized by the subject. Therefore, the operation of others' sad emotion response did not achieve the expected experimental effect[5].

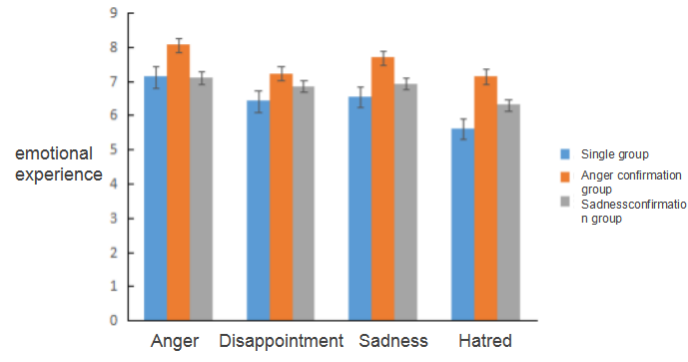

Figure 1 Emotional experience of negative emotional words in different situations 


\section{CONCLUSION}

Although we have tried our best to arrange the subjects to watch both the video and the expression of the fake subjects, due to the limitations of objective conditions, we only effectively manipulated the anger response of others in the group and did not effectively manipulate the sadness response of others. There may be two reasons. First, the attribute of sadness itself is special, depressed, gentle, and arousing. Sad expressions are difficult to recognize and easily confused with other expressions. Therefore, subjects cannot clearly recognize the emotional state of false subjects. Second, around the square table, we set subjects to sit opposite two false subjects, and the video is played in front of the square table. False subject 2 sits diagonally opposite the real subject and compared with false subject 3 . It is more difficult to recognize the expression of false subject 2 . Therefore, only 10 of the 30 subjects were aware of the sadness of false subject 2, but 20 subjects were aware of the sadness of false subject 3. Therefore, in the group situation of the three, the subjects could not fully judge the emotional state of others.

We assume that when individuals share emotional events with others, they will evaluate the emotional response of others. If their emotions are confirmed by others, the individual's emotional experience will be amplified. Because it is necessary to infer the emotional state of others from external behavior, the clue of emotional confirmation is very key. We set up false subjects to better manipulate the emotional response of others, data table When others express their "anger and hatred", the subjects' "anger and hatred "The emotional experience was significantly higher than that of the single group, indicating that compared with the individual experiencing emotional events alone, when the individual shares emotional events with others, the individual's subjective emotional experience is confirmed by others, resulting in the amplification effect of group emotion. Because the subjects were not obviously aware of the expression of others' sadness emotion, the individual sadness emotion of the sadness confirmation group was not confirmed by the group According to the confirmation of others, the sadness experience of the sadness confirmation group is not significantly higher than that of the single group and cannot produce the amplification effect of group emotion [6]. The video materials that induce emotion can induce a variety of negative emotions of subjects. After combining the four negative emotion words and calculating the average, it is found that there are significant differences among the three groups, and the anger confirmation group is significantly higher than that of the single group, indicating that when other members of the group clearly expressed their "anger and hatred" emotions, individuals not only experienced stronger "anger and hatred" emotions, but also experienced more negative emotions. Under the three situational conditions, there was no significant difference in the scores of subjects on each emotional word. However, we can find that the anger and hatred of the anger confirmation group are significantly higher than that of the single group, indicating that the individual's anger and hatred have been confirmed by others to a certain extent, so the experience is higher, which supports our hypothesis to a certain extent.

\section{REFERENCES}

[1] Bauer D J, Sterba S K, Hallfors D D. Evaluating group-based interventions when control participants are ungrouped[J]. Multivariate Behavioural Research, 2008, 43(2): 210-236. DOI: https://doi.org/10.1080/00273170802034810

[2] Fischer, A. H., Rottefella, M. , Evers, C. , \& Manstead, A. . (2004). Emotional assimilation: how we are influenced by others' emotions. Cahiers De Psychologies Cognitive, 22(2), 223-245.

[3] Bruder M, Dosmukhambetova D, Nerb J, et al. Emotional signals in nonverbal interaction: Dyadic facilitation and convergence in expressions, appraisals, and feelings[J]. Cognition \& emotion, 2012, 26(3): 480-502.DOI: https://doi.org/10.1080/02699931.2011.645280

[4] Gonzaga G C, Campos B, Bradbury T. Similarity, convergence, and relationship satisfaction in dating and married couples[J]. Journal of personality and social psychology, 2007, 93(1): 34. DOI: https://doi.org/10.1037/0022-3514.93.1.34

[5] Gordian E H, Yzerbyt V, Wigboldus D, et al. Emotional reactions to harmful intergroup behaviour[J]. European Journal of Social Psychology, 2006, 36(1): 15-30. DOI: https://doi.org/10.1002/ejsp.296

[6] Ivies R, Wagner D T, Morgeson F P. Explaining affective linkages in teams: individual differences in susceptibility to contagion and individualismcollectivism[J]. Journal of applied psychology, 2007, 92(4): 1140 . 